\title{
Aggregation in Charged Nanoparticles Solutions Induced by Different Interactions
}

\author{
S. Abbas ${ }^{1}$, Sugam Kumar ${ }^{1}$, V. K. Aswal ${ }^{1 *}$ and J. Kohlbrecher ${ }^{2}$ \\ ${ }^{1}$ Solid State Physics Division, Bhabha Atomic Research Centre, Mumbai 400 085, India \\ ${ }^{2}$ Laboratory for Neutron Scattering, Paul Scherrer Institut, CH-5232 PSI Villigen, Switzerland \\ vkaswal@barc.gov.in
}

\begin{abstract}
Small-angle neutron scattering (SANS) has been used to study the aggregation of anionic silica nanoparticles as induced through different interactions. The nanoparticle aggregation is induced by addition of salt $(\mathrm{NaCl})$, cationic protein (lysozyme) and non-ionic surfactant (C12E10) employing different kind of interactions. The results show that the interaction in presence of salt can be explained using DLVO theory whereas non-DLVO forces play important role for interaction of nanoparticles with protein and surfactant. The presence of salt screens the repulsion between charged nanoparticles giving rise to a net attraction in the DLVO potential. On the other hand, strong electrostatic attraction between nanoparticle and oppositely charged protein leads to protein-mediated nanoparticle aggregation. In case of non-ionic surfactant, the relatively long-range attractive depletion interaction is found to be responsible for the particle aggregation. Interestingly, the completely different interactions lead to similar kind of aggregate morphology. The nanoparticle aggregates formed are found to have mass fractal nature having a fractal dimension $(\sim 2.5)$ consistent with diffusion limited type of fractal morphology in all three cases.
\end{abstract}

Keywords: Colloids, silica nanoparticles, fractal aggregate, SANS.

PACS: 82.70.Dd, 81.07.-b, 83.85.Hf, 87.15. nr

\section{INTRODUCTION}

The understanding of colloidal aggregation is of high scientific interest because of its multitude applications in the fields ranging from molecular biology to food industry [1]. In general, colloids are stabilized by repulsive interactions (e.g. electrostatic or steric repulsion) against attractive van der Waals forces [2]. Colloidal particles aggregate when physical processes bring particles in contact with each other and thermodynamic stability requires the particle-particle attachment [3]. This can be achieved by modifications in interparticle interactions through addition of selective additives leading to particle aggregation. For example, the presence of electrolyte screens the electrostatic repulsion between the charged stabilized particles which ultimately results into particle aggregation whereas addition of oppositely charged macromolecules causes nonspecific associations giving rise to macromolecule mediated particle aggregation. The morphology of the aggregates thus formed depends on the properties of the particles, solution conditions as well as characteristics of additive, if any.
In the present work, we have studied the evolution of attractive interactions and resultant particle aggregation through different mechanisms. Charge stabilized anionic silica nanoparticles have been used as model colloidal system due to their long term stability and the ability to interact with macromolecules. The nanoparticle aggregation has been induced by adding salt $(\mathrm{NaCl})$, cationic protein (lysozyme) and non-ionic surfactant (C12E10). SANS has been used to characterize these systems as it can provide information on both interaction and resultant structures. Moreover, SANS due to easy possibility of contrast variation is an ideal technique to study such multi component systems $[4,5]$.

\section{EXPERIMENTAL}

Electrostatically stabilized colloidal suspensions of silica nanoparticles (Ludox LS30) and additives [salt $\mathrm{NaCl}$, protein lysozyme and surfactant decaoxyethylene n-dodecylether (C12E10)] were purchased from Sigma-Aldrich. Samples were prepared by dissolving $1 \mathrm{wt} \%$ amount of silica nanoparticles and varying amount of additives in mixed $\mathrm{H}_{2} \mathrm{O} / \mathrm{D}_{2} \mathrm{O}$ solvent as per contrast requirement. Small-angle neutron scattering experiments were 
performed at SANS-I facility, Paul Scherrer Institut, Switzerland. Corrections were made for background and empty cell contributions and data were normalized to absolute cross-sectional unit using standard procedures.

\section{SANS ANALYSIS}

In SANS, one measures the coherent scattering intensity as a function of $Q[\mathrm{Q}=(4 \pi \sin \theta) / \lambda ; 2 \theta$ is scattering angle, $\lambda$ is wave length of neutrons] which can be given by [6]

$$
I(Q)=n V^{2}\left(\rho_{p}-\rho_{s}\right)^{2} P(Q) S(Q)+B
$$

where $n$ is the number density of particles and $V$ is particle volume. $\rho_{p}$ and $\rho_{\mathrm{s}}$ are scattering length densities of particle and solvent, respectively. $P(Q)$ is form factor and $S(Q)$ is interparticle structure factor. $B$ is a constant term representing incoherent background. The expressions of $\mathrm{P}(\mathrm{Q})$ for different shapes are available in literature [6]. The interaction between the particles may be a combination of attractive and repulsive forces. In this case, $\mathrm{S}(\mathrm{Q})$ may be calculated by considering following two-Yukawa (2Y) potential having four parameters $\left(\mathrm{K}_{1}, \mathrm{~K}_{2}, \mathrm{Z}_{1}\right.$ and $\left.\mathrm{Z}_{2}\right)$ [7] :

$$
\begin{aligned}
U(r) & =\infty \text { for } 0<r<\sigma \\
& =-K_{1} \frac{\exp \left\{-Z_{1}(r / \sigma-1)\right\}}{r / \sigma}+K_{2} \frac{\exp \left\{-Z_{2}(r / \sigma-1)\right\}}{r / \sigma} \text { for } r>0
\end{aligned}
$$

where $\sigma$ is particle diameter. $K$ (in units of $k_{B} T, k_{B}$ is Boltzmann constant and $T$ is temperature) is proportional to magnitude of the potential while $1 / Z$ is proportional to the range of the potential. $S(Q)$ for particle aggregation modeled by a mass fractal is given by [6]:

$$
S_{f}(Q)=1+\frac{1}{\left(Q R_{b}\right)^{D}} \frac{D \Gamma(D-1)}{\left[1+\frac{1}{(Q \xi)^{2}}\right]^{(D-1) / 2}} \sin \left\{(D-1) \times \tan ^{-1}(Q \xi)\right\}
$$

where $\xi$ signifies the maximum length up to which fractal microstructure exists, $R_{b}$ is the size of building block and $D$ is the fractal dimension.

\section{RESULTS AND DISCUSSION}

Fig. 1 shows the SANS data of $1 \mathrm{wt} \%$ pure nanoparticles, pure protein $(\mathrm{pH} 7)$ and surfactant in $\mathrm{D}_{2} \mathrm{O}$. The nanoparticle system shows a correlation peak at about $\left(Q \sim 0.1 \mathrm{~nm}^{-1}\right)$ indicating the presence of repulsive interaction between nanoparticles. The data are fitted employing a $P(Q)$ for polydispersed spheres and $S(Q)$ as calculated by Hayter and Penfold analysis under Mean Spherical Approximation for charged macroions [6]. Unlike nanoparticles, data of surfactant and protein both show monotonically decreasing profile which is a typical feature of form factor governed scattering $(S(Q) \sim 1)$. The protein has been modeled with ellipsoidal shape while surfactant micelles as consisting of a spherical core attached with Gaussian chains. The charge on the protein (Table 1) is determined from the concentrated sample ( $5 \mathrm{wt} \%$ ), where $\mathrm{S}(\mathrm{Q})$ contribution is observed (inset of Fig. 1).

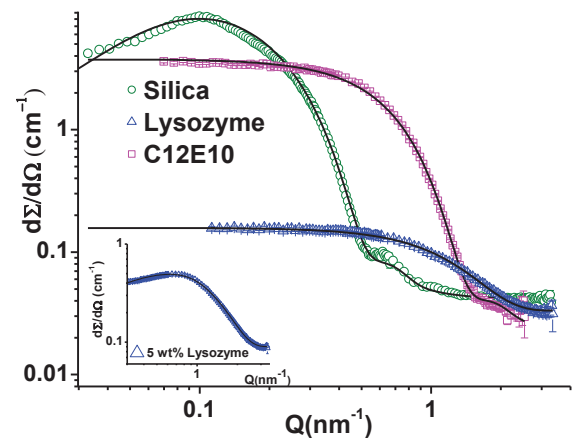

FIGURE 1. SANS data of $1 \mathrm{wt} \%$ silica nanoparticles, lysozyme protein and C12E10 micelles each. Inset shows the SANS data of $5 \mathrm{wt} \%$ lysozyme.

TABLE 1. Structural parameters of pure systems.

\begin{tabular}{lccc}
\hline $\begin{array}{c}\text { System } \\
\mathbf{( 1} \text { wt \%) }\end{array}$ & $\begin{array}{c}\text { Semi-major } \\
\text { Axis (nm) }\end{array}$ & $\begin{array}{c}\text { Semi-minor } \\
\text { Axis (nm) }\end{array}$ & $\begin{array}{c}\text { Charge } \\
\text { (e.u.) }\end{array}$ \\
\hline silica & 7.8 & 7.8 & +40 \\
lysozyme & 2.4 & 1.4 & -7 \\
C12E10 & 1.8 & 1.8 & 0 \\
\hline
\end{tabular}

The silica nanoparticles being charged attain long term stability as per DLVO theory where electrostatic repulsion overcomes the van der Waals attraction. The presence of different additives may modify interparticle interactions as a combination of DLVO as well as non-DLVO forces. SANS data of $1 \mathrm{wt} \%$ silica nanoparticles with different amount of three additives ( $\mathrm{NaCl}$, lysozyme and $\mathrm{C} 12 \mathrm{E} 10$ ) in $\mathrm{H}_{2} \mathrm{O} / \mathrm{D}_{2} \mathrm{O}$ mixed solvent for which micelles and proteins are contrastmatched are shown in Fig. 2. The SANS data show increase in the low Q intensity indicating the evolution of the attractive interaction in the system. The data have been analyzed by considering $\mathrm{S}(\mathrm{Q})$ as calculated from a two-Yukawa potential accounting for both attractive as well as repulsive interactions between nanoparticles. The fitted potentials are shown in Fig. 3. The significantly different amounts of additives are required to provoke similar strength of the overall attraction between the nanoparticles. This is because of the different mechanisms responsible for the evolution of attraction in the system. In case of electrolyte, it screens the electrostatic repulsion between the charged nanoparticles which induces a net attraction. The total interaction potential in this case can be understood as a DLVO potential combining van der Waals attraction with a weak screened Coulomb repulsion. The presence of protein molecules mediates a strong but 
short-range attraction between two otherwise repelling nanoparticles [4]. The range of this attraction is determined by the size of the protein molecule. On the other hand, non-ionic micelles cause relatively longrange depletion attraction between nanoparticles [5].

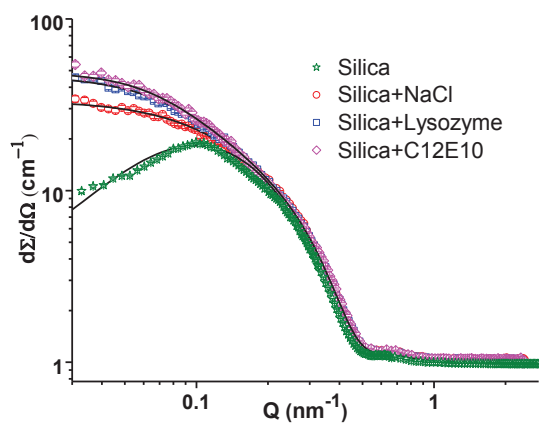

FIGURE 2. SANS data of $1 \mathrm{wt} \%$ silica nanoparticles mixed with $\mathrm{NaCl}(0.4 \mathrm{M})$, lysozyme $(0.01 \mathrm{wt} \%)$ and $\mathrm{C} 12 \mathrm{E} 10$ $(0.01 \mathrm{wt} \%)$

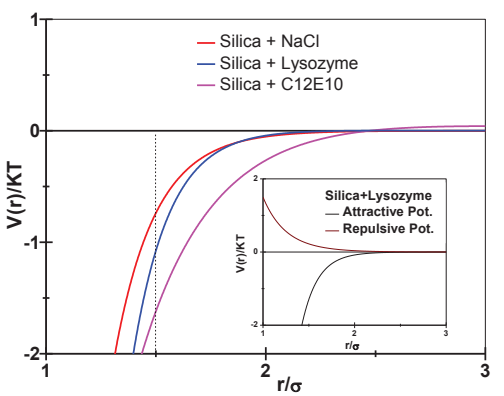

FIGURE 3. Fitted total interaction potential corresponding to SANS data shown in Fig. 2. The vertical line represents average distance between the particles. Inset shows calculated attractive and repulsive parts of the total potential in silica+lysozyme system.

The strength of the attraction increases with increase in the additive concentration in all three cases leading to nanoparticle aggregation. Though, the mechanisms of the particle aggregation are completely different but lead to similar kind of nanoparticle aggregates. The SANS data of $1 \mathrm{wt} \%$ silica nanoparticles with higher amount of additives have been shown in Fig. 4. The linearity in the low Q region suggests the fractal nature of the aggregates. Since no low Q cut off is seen in the data within Q range of our measurements so a value more than $2 \pi / \mathrm{Q}_{\min }$ for $\xi$ (Eqn. 3 ) has been used during the analysis. The nanoparticle aggregates are found to have mass fractal nature with almost same fractal dimension (2.5) [Table 2]. A fractal dimension about 2.5 indicates a diffusion limited aggregate (DLA) type of fractal morphology. These fractals are known to form in the low particle density interacting with relatively weak repulsive forces [8].

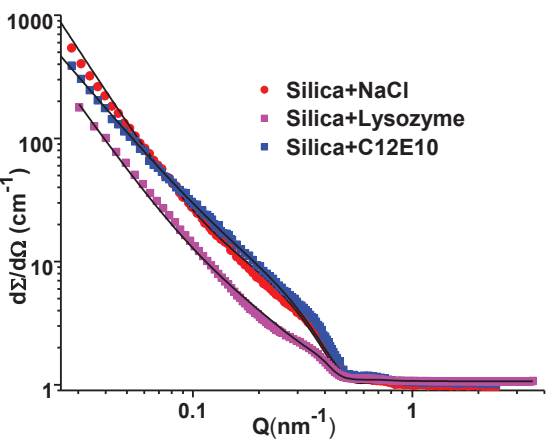

FIGURE 4. SANS data of $1 \mathrm{wt} \%$ silica nanoparticles mixed with $\mathrm{NaCl}(2.0 \mathrm{M})$, lysozyme $(0.05 \mathrm{wt} \%)$ and $\mathrm{C} 12 \mathrm{E} 10(0.1$ $w t \%)$.

TABLE 2. Structural parameters of nanoparticle aggregates as formed by different additives.

\begin{tabular}{lcc}
\hline Additive & $\begin{array}{c}\text { Building Block } \\
\text { Radius (nm) }\end{array}$ & $\begin{array}{c}\text { Fractal } \\
\text { Dimension }\end{array}$ \\
\hline $\mathrm{NaCl}$ & 7.8 & 2.6 \\
lysozyme & 7.8 & 2.5 \\
$\mathrm{C} 12 \mathrm{E} 10$ & 7.8 & 2.2 \\
\hline
\end{tabular}

\section{CONCLUSIONS}

SANS has been used to understand the mechanism of aggregation of silica nanoparticles by addition of electrolyte, cationic protein and nonionic surfactant. The interaction in presence of salt has been explained using DLVO theory where screening of electrostatic repulsion by salt ions gives rise to a net attraction which finally results in particle aggregation with increasing salt concentration. The presence of oppositely charged protein mediates the conjugation of nanoparticles through electrostatic complexion. In case of non-ionic surfactant, it induces attractive depletion between nanoparticles causing particle aggregation. Moreover, completely different mechanisms lead to similar kind of fractal structure on nanoparticle aggregates.

\section{REFERENCES}

1. R. Mezzenga, P. Schurtenberger, A. Burbidge and M. Michel, Nature Materials 4, 729 (2005).

2. C. N. Likos, Phys. Rep. 348, 267-439 (2001).

3. E. M. Hotze, T. Phenrat and G. V. Lowry, J. Environ. Qual. 39, 1909- 1924 (2010).

4. Sugam Kumar, V. K. Aswal and J. Kohlbrecher, Langmuir 27, 10167 (2011)

5. Sugam Kumar, M.-J. Lee, V. K. Aswal, and S.-M. Choi, Phys. Rev. E 87, 042315 (2013).

6. J. S. Pedersen, Adv. Coll. Inter. Sci. 70, 171-210 (1997).

7. Y. Liu, W.-R. Chen, and S.-H. Chen, J. Chem. Phys. 122, 044507 (2005).

8. T.A Witten and L.M Sander, Phys. Rev. Lett. 47 14001403 (1981). 